\title{
Relative Equilibrium Plot Improves Graphical Analysis and Allows Bias Correction of Standardized Uptake Value Ratio in Quantitative ${ }^{11} \mathrm{C}$-PiB PET Studies
}

\author{
Yun Zhou ${ }^{1}$, Jitka Sojkova ${ }^{1,2}$, Susan M. Resnick ${ }^{2}$, and Dean F. Wong ${ }^{1}$ \\ ${ }^{I}$ Russell H. Morgan Department of Radiology and Radiological Science, School of Medicine, Johns Hopkins University, Baltimore, \\ Maryland; and ${ }^{2}$ Laboratory of Behavioral Neuroscience, Intramural Research Program, National Institute on Aging, National \\ Institutes of Health, Baltimore, Maryland
}

Both the standardized uptake value ratio (SUVR) and the Logan plot result in biased distribution volume ratios (DVRs) in ligandreceptor dynamic PET studies. The objective of this study was to use a recently developed relative equilibrium-based graphical (RE) plot method to improve and simplify the 2 commonly used methods for quantification of ${ }^{11} \mathrm{C}$-Pittsburgh compound $\mathrm{B}\left({ }^{11} \mathrm{C}\right.$ PiB) PET. Methods: The overestimation of DVR in SUVR was analyzed theoretically using the Logan and the RE plots. A biascorrected SUVR (bcSUVR) was derived from the RE plot. Seventyeight ${ }^{11} \mathrm{C}-\mathrm{PiB}$ dynamic PET scans (66 from controls and 12 from participants with mild cognitive impaired [MCl] from the Baltimore Longitudinal Study of Aging) were acquired over $90 \mathrm{~min}$. Regions of interest (ROls) were defined on coregistered MR images. Both the $\mathrm{ROI}$ and the pixelwise time-activity curves were used to evaluate the estimates of DVR. DVRs obtained using the Logan plot applied to ROI time-activity curves were used as a reference for comparison of DVR estimates. Results: Results from the theoretic analysis were confirmed by human studies. ROI estimates from the RE plot and the bcSUVR were nearly identical to those from the Logan plot with ROI time-activity curves. In contrast, ROI estimates from DVR images in frontal, temporal, parietal, and cingulate regions and the striatum were underestimated by the Logan plot (controls, 4\%-12\%; MCI, 9\%-16\%) and overestimated by the SUVR (controls, $8 \%-16 \%$; MCI, 16\%-24\%). This bias was higher in the $\mathrm{MCl}$ group than in controls $(P<0.01)$ but was not present when data were analyzed using either the RE plot or the bcSUVR. Conclusion: The RE plot improves pixelwise quantification of ${ }^{11} \mathrm{C}-\mathrm{PiB}$ dynamic $\mathrm{PET}$, compared with the conventional Logan plot. The bcSUVR results in lower bias and higher consistency of DVR estimates than of SUVR. The RE plot and the bcSUVR are practical quantitative approaches that improve the analysis of ${ }^{11} \mathrm{C}-\mathrm{PiB}$ studies.

Key Words: RE plot; ${ }^{11} \mathrm{C}-\mathrm{PiB}$; PET; SUVR; bias

J Nucl Med 2012; 53:622-628

DOI: 10.2967/jnumed.111.095927

\footnotetext{
Received Aug. 30, 2011; revision accepted Dec. 12, 2011.

For correspondence contact: Yun Zhou, Russell H. Morgan Department of Radiology and Radiological Science, Johns Hopkins University School of Medicine, 601 N. Caroline St., JHOC Room 3245, Baltimore, MD 21287-0807. E-mail: yunzhou@jhmi.edu

Published online Mar. 13, 2012.

COPYRIGHT ( 2012 by the Society of Nuclear Medicine, Inc.
}

$\mathbf{P}$ ittsburgh compound B labeled with ${ }^{11} \mathrm{C}\left({ }^{11} \mathrm{C}-\mathrm{PiB}\right)$ is one of the most widely used compounds for in vivo detection of fibrillar $\beta$-amyloid deposition in brain (1). The spatial distribution of amyloid deposition in Alzheimer disease, mild cognitive impairment (MCI), and adults who are cognitively normal has been well characterized (2-5). The distribution volume ratio (DVR) or binding potential (DVR - 1) is usually used as an index of ${ }^{11} \mathrm{C}-\mathrm{PiB}$-specific binding to tissue for quantitative measurement of $\beta$-amyloid deposition (6). In ligand-receptor dynamic PET studies, DVR can be estimated by fitting a kinetic model to the tissue kinetics with a metabolite-corrected plasma input function $(7,8)$, where the tracer kinetic model can be a classic compartment model or a model-independent graphical analysis method and the plasma input function is the tracer radioactivity in plasma, usually measured by serial arterial blood sampling. However, quantitative PET without arterial blood sampling is more practical, and various reference tissue models have been evaluated for noninvasive quantification of ${ }^{11} \mathrm{C}-\mathrm{PiB}$ dynamic PET (9-14). Importantly, in these studies, the target-to-reference (cerebellum) tissue tracer concentration ratio was shown to achieve a constant at approximately 50 min after tracer injection $(12,13)$, lending itself to pixelwise analysis using simplified, noncompartmental approaches (14). Many ${ }^{11} \mathrm{C}-\mathrm{PiB}$ studies have used standardized uptake value ratio (SUVR) with a reference tissue because of this method's simplicity and short scan time, in which the SUVR is calculated as target-toreference tissue tracer concentration ratio over time frame (T0 T1) after tracer injection, where T0 and $\mathrm{T} 1$ are the start and end times of PET scanning.

Although there are variations in the selection of time frames (T0 T1) for SUVR measurements (15), the main disadvantage of the SUVR is the positive bias resulting in overestimation of DVR $(16,17)$. In contrast, a commonly used Logan plot demonstrates inherent negative bias, which is attributed to noise and the target tissue tracer concentration (18-22). The linearized methods, particularly the multilinear reference tissue model (23), provide estimates of DVR similar to those from a simplified reference tissue model (SRTM) 
(9) yet with much higher computational efficacy. At the pixel level, PET measurements have high noise, and thus underestimation of DVR occurs with both graphical (19) and linearized methods $(10,18,24)$. Although all of these approaches are associated with bias, and bias may be one of the limiting factors for application of the simplified quantitative methods to this tracer, bias consistency across clinical groups has not been extensively explored in ${ }^{11} \mathrm{C}-\mathrm{PiB}$ studies.

A novel, noninvasive, and computationally efficient graphical approach, the relative equilibrium-based graphical (RE) plot, has been recently developed for analysis of tracer kinetics, with equilibrium relative to input function (25). For reference tissue input function, target tissue tracer kinetics attain a relative equilibrium if there is a $t^{*}$ such that the ratio of the target to reference tissue tracer concentration is constant for $\mathrm{t} \geq \mathrm{t} *$. In this study, the RE plot with reference tissue input was used to analyze the overestimation of DVR in SUVR measurements, develop a practical method that allows correction of the overestimation bias in SUVR, and generate DVR images that avoid the noise-induced underestimation in estimates from the Logan plot.

\section{MATERIALS AND METHODS}

\section{Correction of DVR Overestimation in SUVR Using RE Plot with Reference Tissue Input}

The inconsistent overestimation of DVR in SUVR was first demonstrated using the Logan plot with plasma input (supplemental data, available online only at http://jnm.snmjournals.org). In this section, the RE plot with reference tissue input is used to analyze the overestimation of DVR in SUVR. Let $\mathrm{C}(\mathrm{t})$ and $\mathrm{C}_{\mathrm{REF}}(\mathrm{t})$ be the tracer concentrations at time $t$ in target and reference tissues, respectively. In this study, we assume that there is a time $t^{*}$ such that the tissue kinetics attain equilibrium relative to the reference tissue input- that is, $C(t) / C_{R E F}(t)$ is a constant for $t \geq t *$-and reference tissue input $C_{R E F}(t)$ can be approximated by 1 exponential as $C_{R E F}(t)=\alpha e^{\beta t}$ for $t \geq t^{*}$. For tracer kinetics that attain relative equilibrium, the $R E$ plot with reference tissue input (25) described by Equation 1 has been proposed to simplify and improve the generation of DVR images using the conventional Logan plot with reference tissue input:

$$
\frac{\int_{0}^{t} \mathrm{C}(\mathrm{s}) \mathrm{ds}}{\mathrm{C}_{\mathrm{REF}}(\mathrm{t})}=\operatorname{DVR} \frac{\int_{0}^{\mathrm{t}} \mathrm{C}_{\mathrm{REF}}(\mathrm{s}) \mathrm{ds}}{\mathrm{C}_{\mathrm{REF}}(\mathrm{t})}+\theta,
$$

where DVR is the slope of the linear portion of the plots for $t \geq t^{*}$, and $\theta$ is the $y$-intercept of the linear regression. It has been shown that the RE plot is an unbiased graphical analysis method for estimation of DVR with no noise-induced underestimation of the DVR estimates (25).

By taking derivative of both sides of Equation 1 in bilinear form and then dividing by $\mathrm{C}_{\mathrm{REF}}(\mathrm{t})$, we have

$$
\frac{\mathrm{C}(\mathrm{t})}{\mathrm{C}_{\mathrm{REF}}(\mathrm{t})}=\mathrm{DVR}+\theta \frac{\mathrm{C}_{\mathrm{REF}}^{\prime}(\mathrm{t})}{\mathrm{C}_{\mathrm{REF}}(\mathrm{t})},
$$

where $\mathrm{C}_{\text {REF }}^{\prime}(\mathrm{t})$ is the derivative of $\mathrm{C}_{\mathrm{REF}}(\mathrm{t}), \mathrm{C}(\mathrm{t}) / \mathrm{C}_{\mathrm{REF}}(\mathrm{t})$ is a constant that is defined as SUVR, and $\mathrm{C}_{\text {REF }}^{\prime}(\mathrm{t}) / \mathrm{C}_{\mathrm{REF}}(\mathrm{t})=\beta$ for $\mathrm{t} \geq \mathrm{t}^{*}$.
Therefore, the relationship between SUVR and DVR is expressed by Equation 3:

$$
\mathrm{SUVR}=\mathrm{DVR}+\theta \beta
$$

For the tracer delivered by bolus injection, $\beta$ is negative. The $\theta$ in the RE plot is also negative, and its absolute value is positively correlated with the DVR (25). Therefore, the bias term $\theta \beta$ in Equation 3 is positive, and the overestimation of DVR in SUVR increases as DVR increases.

Equation 3 shows a linear relationship between $\theta$ and SUVR at a given $\beta$. We can rewrite the linear relationship between $\theta$ and SUVR as

$$
\theta=\lambda \mathrm{SUVR}+\mu, \quad \text { Eq. } 4
$$

where $\lambda$ and $\mu$ are constants across all subjects. On the basis of Equation 4, the bcSUVR of estimate of DVR can be calculated using $\lambda, \mu$, and $\beta$ as Equation 5:

$$
\text { bcSUVR }=\text { SUVR }-(\lambda \operatorname{SUVR}+\mu) \beta . \quad \text { Eq. } 5
$$

In Equation 5, $\beta$ was estimated by fitting 1 exponential to the reference tissue input $C_{\text {REF }}(t)$ for $t \geq t^{*}$ using linear regression of time $t$ (independent regression variable) versus the natural logarithm of $\mathrm{C}_{\mathrm{REF}}(\mathrm{t})$ (dependent regression variable) for each dynamic PET scan.

For the region-of-interest (ROI)-based bias correction of SUVR, the ROI-specific $\lambda$ and $\mu$ were estimated by linear regression using Equation 4 with $\theta$ and SUVR from all subjects by assuming that Equation 4 holds for the whole population for a given ROI. The ROI bcSUVR for each subject was then calculated using Equation 5 with the ROI-specific $\lambda$ and $\mu$ from the population and $\beta$ from the subject's $C_{\text {REF }}(t)$.

For pixelwise bias correction of SUVR, Equation 6 was used to generate bcSUVR images for each subject:

$$
\mathrm{bcSUVR}=\mathrm{SUVR}-\left(\lambda_{\mathrm{m}} \mathrm{SUVR}+\mu_{\mathrm{m}}\right) \beta, \quad \text { Eq. } 6
$$

where $\lambda_{\mathrm{m}}=\left(\sum_{\mathrm{i}=1}^{\mathrm{M}} \lambda_{\mathrm{i}}\right) / \mathrm{M}$ and $\mu_{\mathrm{m}}=\left(\sum_{\mathrm{i}=1}^{\mathrm{M}} \mu_{\mathrm{i}}\right) / \mathrm{M}$, the ROI-specific $\lambda_{\mathrm{i}}$ and $\mu_{i}$ are constants for all subjects obtained as described for ROI-based bias correction, and $\mathrm{M}$ is the sample size of the population including both controls and MCI subjects. As in Equation $5, \beta$ was estimated from reference tissue input function $C_{R E F}(t)$ $\left(t \geq t^{*}\right)$ for each subject. The simplified pixelwise bias-correction of the SUVR method is based on the following observations: low variation of ROI $\lambda$ s (Table 1$)$ and mean $($ bcSUVR) $=$ mean $\left(\right.$ SUVR) $-\lambda_{\mathrm{m}} \operatorname{mean}(\beta S U V R)-\mu_{\mathrm{m}} \operatorname{mean}(\beta)$, where the bias correction term $\mu_{\mathrm{m}}$ mean $(\beta)$ remains constant in control and patient groups because the mean $(\beta)$ in each group is usually the samethat is, $\mu_{m} \beta$ does not contribute to the correction of inconsistent overestimation of DVR in SUVR. In other words, $\mu_{m}$ could be biased, but the $\mu_{\mathrm{m}}$-induced bias to bcSUVR contributed to each group is the same.

\section{Human ${ }^{11} \mathrm{C}-\mathrm{PiB}$ Dynamic PET}

Study Participants. Seventy-eight ${ }^{11} \mathrm{C}-\mathrm{PiB}$ dynamic PET studies of nondemented older participants in the neuroimaging substudy of the Baltimore Longitudinal Study on Aging (26) were acquired. In conjunction with each ${ }^{11} \mathrm{C}-\mathrm{PiB}$ study, all participants received a detailed physical examination, including medical history updates 


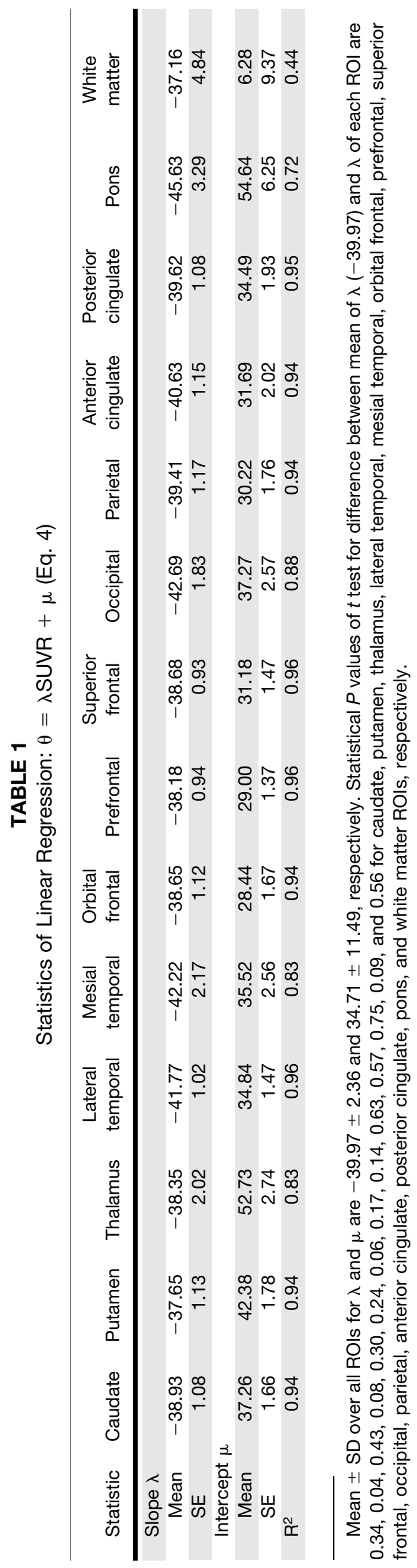

and laboratory screening, neuropsychologic testing, and assessment by the Clinical Dementia Rating (CDR) (27) scale. The CDR scores were typically based on informant (spouse, child, or close friend) interviews conducted by a certified examiner. Cognitive status was determined by consensus diagnosis according to established procedures $(28,29)$. A diagnosis of MCI was made in participants who had cognitive impairment (typically memory) but did not have functional loss in activities of daily living. Sixty-six scans from individuals with a CDR of 0 (age range at baseline scan, 55.7-92.1 y; mean age $\pm \mathrm{SD}, 77.6 \pm 6.9$ y) were classified as the control group, and 12 scans from individuals with a CDR of 0.5 (age range at baseline scan, 77.2-89.5 y; mean age \pm SD, $83.5 \pm 4.3 \mathrm{y}$ ) were classified as the MCI group. In this study, we considered a CDR of 0.5 as indicative of MCI, but only 3 individuals actually met the consensus criteria for MCI.

${ }^{11}$ C-PiB Dynamic PET. All dynamic PET studies were obtained on an Advance scanner (GE Healthcare). PET was started immediately after an intravenous bolus tracer injection (543.5 \pm 29.7 MBq; range, 442.9-605.3 MBq) with a high specific activity $(236.6 \pm 145.4 \mathrm{GBq} / \mu \mathrm{mol}$; range, $36.1-1,005.4 \mathrm{GBq} / \mu \mathrm{mol})$ at the time of injection of ${ }^{11} \mathrm{C}$-PiB. There were no significant differences in ${ }^{11} \mathrm{C}$-PiB dose or specific activity among cognitively impaired and cognitively normal older adults $(P>0.05)$. Dynamic PET data were collected in 3-dimensional acquisition mode according to the following protocol: $4 \times 0.25,8 \times 0.5,9 \times 1,2 \times 3$, and $14 \times$ $5 \mathrm{~min}$ (90 min total, 37 frames). To minimize head motion during PET, all participants were fitted with thermoplastic face masks. The reconstruction of dynamic PET images was described in our previous studies (10).

Structural MRI and ROI Definition. Structural MR images for anatomic localization were obtained on a Signa 1.5-T (GE Healthcare) or Intera 3.0-T (Phillips) scanner using a spoiled gradient-recalled acquisition sequence (124 slices with a $256 \times$ 256 image matrix, pixel size of $0.94 \times 0.94 \mathrm{~mm}$, and slice thickness of $1.5 \mathrm{~mm}$ ). MR images were typically obtained on the same visit, but a major renovation of the MRI research scanners coincided with ${ }^{11} \mathrm{C}-\mathrm{PiB}$ imaging studies. Although $61 \mathrm{MR}$ images were acquired at the time of the PET scan, 15 were obtained within $1.8 \mathrm{y}$ (SD, $0.8 \mathrm{y}$; range, 1-4.1 y) of the PET scan. Two additional MR images were acquired 10.5 and $11.8 \mathrm{y}$ before the PET scans because of medical issues that prevented concurrent MRI assessments. MR images were coregistered to the mean image of the first 20 min of dynamic PET scanning using SPM2 (Statistical Parametric Mapping software; Wellcome Department of Cognitive Neurology) with the mutual-information method. In addition to the reference region (cerebellar cortex), 14 ROIs were manually drawn on the coregistered MR images $(10,12,13)$ and copied to the dynamic PET images to obtain ROI time-activity curves for kinetic analysis. The ROIs were also applied directly to parametric images for estimation of DVRs.

\section{Evaluation of RE Plot and Parameter Estimation}

The evaluation of assumptions for the RE plot is provided in the supplemental data. The single t* value used in the RE and Logan plots and SUVR (supplemental data) for all subjects were determined at $52.5 \mathrm{~min}$ after tracer injection, corresponding to the last 8 time frames, from 50 to $90 \mathrm{~min}$ of dynamic scanning. DVRs from the RE plot, the Logan plot with reference tissue input $(10,11,25)$ (hereafter, the Logan plot) (supplemental data), and the SUVR were calculated from ROI kinetics. Subsequently, parametric images from pixelwise kinetics were obtained using the RE and 
Logan plots, the SUVR and bcSUVR, and the SRTM. The ROIs from the parametric images were then compared with the DVRs from ROI kinetics. The estimates of DVR from the low noise levels of ROI time-activity curves were used as a reference in this study. Mean parametric images for the control and the MCI groups were then generated using SPM2. The spatial normalization parameters determined by the mean image of the first $20 \mathrm{~min}$ of dynamic PET scanning were applied to all parametric images (10).

All parameter estimation methods for ROI kinetic analysis and parametric image generation were written in MATLAB (The MathWorks Inc.) and implemented on a PWS690 workstation (Dell).

\section{Evaluation of Assumptions for Bias-Corrected SUVR}

The values of $\beta$ obtained by fitting 1 exponential to the reference tissue input $C_{\mathrm{REF}}(\mathrm{t})$ for $\mathrm{t} \geq \mathrm{t}^{*}=52.5$ min were compared between patient and control groups using the statistical $t$ test.

To evaluate the assumption that $\mathrm{C}_{\mathrm{REF}}(\mathrm{t})$ can be approximated by 1 exponential as $C_{\text {REF }}(t)=\alpha e^{\beta t}$ for $t \geq t^{*}=52.5$ min, $C_{R E F}(t)$ was fitted not only by 1 exponential but also by multiexponentials as $\mathrm{C}_{\mathrm{REF}}(\mathrm{t})=\alpha_{0}+\sum^{\mathrm{n}} \alpha_{\mathrm{i}} \mathrm{e}^{-\beta_{\mathrm{i}} \mathrm{t}}$ for $\mathrm{t} \geq \mathrm{t}^{*}=52.5$, where $\alpha_{0}$ is a constant and $\mathrm{n}$ is the number of exponentials, and along with $\left\{\left(\alpha_{\mathrm{i}}, \beta_{\mathrm{i}}\right) \mid 1 \leq \mathrm{i} \leq \mathrm{n}\right\}$ were estimated simultaneously by spectral analysis (30-32). To compare the goodness of fit between 1exponential and multiexponential model fitting, the Akaike information criterion (AIC) $(16,33,34)$ was calculated. A model associated with the lower Akaike information criterion is considered the better fitting.

The assumption of the linear relationship between $\theta$ and SUVR was evaluated by applying linear regression of $\theta$ ( $y$-variable) versus SUVR ( $x$-variable) for each ROI time-activity curve over all subjects, where $\theta$ was estimated by the RE plot with $t^{*}=$ $52.5 \mathrm{~min}$

\section{RESULTS}

\section{Validation of Assumptions for Bias-Corrected SUVR}

We verified the 2 assumptions used to derive the bcSUVR from the RE plot. The first assumption is that 1exponential clearance occurs after $t^{*}$ for reference tissue (cerebellum) kinetics. The cerebellum time-activity curves were well fitted by an exponential function at $\mathrm{t} \geq \mathrm{t}^{*}=$ $52.5 \mathrm{~min}$. The $R^{2}$ of the linear regression of time $\mathrm{t}$ versus the natural logarithm of cerebellum time-activity curves was $0.790 \pm 0.196$ and $0.867 \pm 0.071$ for controls and the MCI group, respectively. The $\beta$-values, the 1 -exponential clearance rates of tracer concentration in cerebellum, were $0.007 \pm 0.003 \mathrm{~min}^{-1}$ in controls and $0.008 \pm 0.002$ in the MCI group. They were not significantly different between these 2 groups $(P=0.33)$. The value of the Akaike information criterion calculated from 1-exponential fitting $(-87.47 \pm 6.34)$ was significantly lower than that obtained from multiexponential fitting $(-85.88 \pm 6.98$; paired $t$ test, $P=0.009$ ), and the results demonstrate that the 1-exponential model better predicts or fits reference tissue input $\mathrm{C}_{\mathrm{REF}}(\mathrm{t})$ for $\mathrm{t} \geq \mathrm{t}^{*}=52.5 \mathrm{~min}$.

The second assumption is that there is a linear relationship between SUVR and $\theta$. As shown for a representative ROI, the posterior cingulate cortex, there was a highly linear relationship between SUVR and bias correction factor $\theta$, where absolute value of the correction factor $\theta$ increases as SUVR increases (supplemental data; Fig. 3). The values for $\lambda$ and $\mu$, that is, the slope and the intercept of the linear regression of $\theta$ versus SUVR, for all 14 ROIs are listed together with $R^{2}$ values in Table 1 . The percentage coefficient of variation $(=100[\mathrm{SD} /$ mean $]$ ) of $\lambda$ was $5.6 \%$. Because of this low variation of $\lambda \mathrm{s}$ in different ROIs, there was no significant difference between mean $\lambda$ and ROI-specific $\lambda$ for any ROIs $(P=0.33 \pm 0.23$; range, $0.06-0.75)$ except the putamen $(P=0.04)$.

\section{Bias and Bias Correction of SUVR in ROI Kinetics}

Inconsistent overestimation of the DVR in SUVR between controls and MCI subjects is illustrated in Figure 1A. The SUVR overestimation in this figure is calculated as percentage bias (Bias\%) relative to the DVR estimated from ROI time-activity curves using the Logan plot. The overestimation of DVRs in SUVR ranges from $16 \%$ to $32 \%$ in the MCI group and is significantly higher (on average 1.73 times higher) than in controls in all but 4 ROIs (mesial temporal, occipital, pons, and white matter).

The bcSUVR was developed to address the inconsistency bias of the SUVR. Figure 1B shows that this method is associated with minimal bias (Bias\% $\leq 2.0 \%$ for all ROIs, except $3.6 \%$ for pons) relative to the DVRs estimated from ROI time-activity curves using the Logan plot and that there is no difference between the MCI group and control group in Bias\% (statistical $P$ value, $0.46 \pm 0.28$; range, 0.08 to 0.94$)$. Figures $2 \mathrm{~A}$ and $2 \mathrm{~B}$ show that, after using the bcSUVR, the linear relationship between the SUVR and DVR from ROI time-activity curves using the Logan plot improved, because the slope of the regression line changed from 1.37 to 1.02 and $R^{2}$ increased from 0.94 to 0.96 .

\section{SUVR, bcSUVR, and DVR Images}

The constants for generating the bcSUVR images, $\lambda$ and $\mu$, are -39.97 and 34.71 , respectively (Table 1 ). The mean images of SUVR, bcSUVR, and DVRs generated by the RE and Logan plots are shown in Figure 3. As expected, there is overestimation in the SUVR images and noise-induced underestimation in the DVR images generated by the Logan plot. Because the ROIs from SUVR images are identical to those from ROI time-activity curves, the overestimation of DVR in SUVR images was the same as shown in Figures $1 \mathrm{~A}$ and $2 \mathrm{~A}$. The bcSUVR images are comparable to the images generated by the RE plot. There was a highly linear correlation between the ROI values from bcSUVR images and the bcSUVR calculated from ROI time-activity curves: (bcSUVR image) $=0.96 \mathrm{DVR}(\mathrm{bcSUVR}$ ROI time-activity curves) +0.05 , with $R^{2}=0.98$. As compared with the DVRs from the RE plot or the Logan plot with ROI timeactivity curves, the Bias\% of bcSUVR was less than $\pm 6 \%$ for ROIs including the cortex, striatum, and pons; approximately $-11 \%$ in the thalamus; and approximately $8 \%$ in white matter. In addition, no significant difference in Bias $\%$ of bcSUVR was observed between the MCI group and 
FIGURE 1. Variability of Bias $\%$ in ROI DVR estimates relative to DVRs from Logan plot with reference tissue input and $\mathrm{ROI}$ time-activity curves between $\mathrm{MCl}$ and control groups: SUVR (A), bcSUVR (B), DVR from parametric images generated by Logan plot (C), and DVR from RE plot (D). Bias $\%=100$ (DVR/DVR(Logan plot with ROI time-activity curves) - 1). Regions of interest (ROIs) are numbered as 1 , caudate; 2, putamen; 3 , thalamus; 4, lateral temporal; 5, mesial temporal; 6 , orbital frontal; 7, prefrontal; 8 , superior frontal; 9, occipital; 10, parietal; 11, anterior cingulate; 12, posterior cingulate; 13 , pons; and 14, white matter. For RE plot, DVRs from ROI time-activity curves are identical to those from DVR images. Although group differences in Bias\% are detected for SUVR and DVR images generated by Logan plot from high-noise-pixel time-activity curves, bcSUVR and DVRs from the RE plot do not show any group differences. ${ }^{\star} 0.02 \leq P \leq 0.05$. ${ }^{\star \star} P<0.01$.

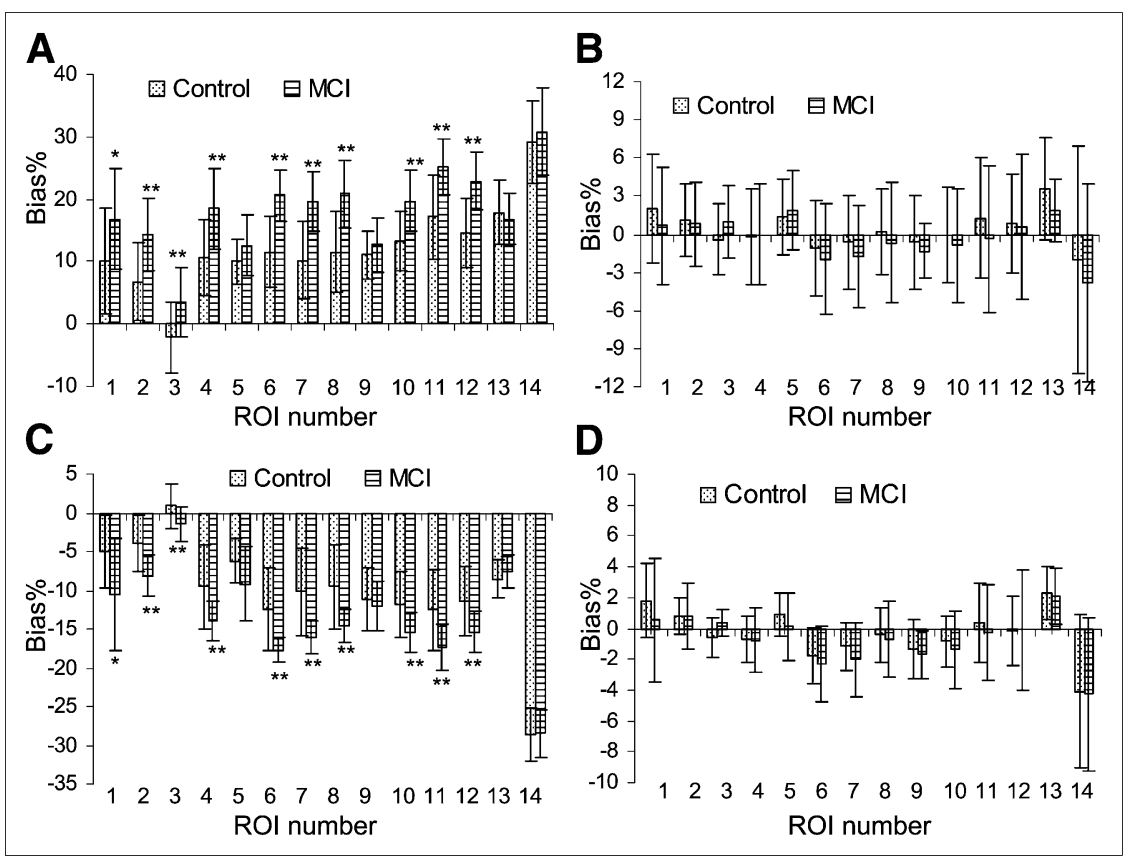

controls. For the Logan plot, the DVR ROI values obtained directly from the parametric DVR images were lower by approximately $12.2 \%$ (excluding white matter) than those from the ROI time-activity curves from low noise levels. The underestimation in the DVR images in the MCI group was significantly higher than that of controls in all but 4 ROIs (mesial temporal, occipital, pons, and white matter) (Fig. 1C). For the RE plot, the DVR estimates from ROI time-activity curves were identical to those obtained by applying ROIs to DVR images. Therefore, the noise-induced underestimation in the DVR images generated by the Logan plot in both control and MCI groups was removed completely using the RE plot (Figs. 1D, 2C, and 2D).

\section{Comparison of DVR Estimates Between Control and $\mathrm{MCl}$ Groups}

The comparison of ROI estimates of DVR between control and MCI groups is summarized in Supplemental Table 2. The DVR estimates from all methods were consistently higher in the MCI group than in controls $(P<0.05)$ for all ROIs except for mesial temporal and occipital cortex, pons, and white matter. In comparison to the DVRs from the Logan plot with ROI time-activity curves, the contrast between DVR in MCI and control groups was increased when quantitated by SUVR and decreased when parametric images were generated by the Logan plot. On the basis of the DVRs estimated from the ROI time-activity curves using the Logan plot, the DVRs in the following ROIs were higher $(P<0.05)$ in the MCI group than in the control group: frontal (orbital, prefrontal, and superior), by $36.1 \%$; cingulate (anterior, and posterior), by $31.7 \%$; parietal, by $20.8 \%$; lateral temporal, by $20.1 \%$; striatum (caudate, putamen), by $26.2 \%$; and thalamus, by $10.6 \%$. In contrast, the difference between MCI and control groups was artificially increased by $8.5 \%$ for SUVR and decreased by $5.6 \%$ for the parametric DVR images generated by the Logan plot. As demonstrated in Supplemental Table 2, for both control and MCI groups, the DVRs estimated by bcSUVR from ROI time-activity curves, bcSUVR images, and RE plot are similar to those from the Logan plot from ROI time-activity curves. The percentage differences in DVRs between MCI and control groups in ROIs (mesial temporal, pons, and white matter) were consistent across all methods, with less than $6 \%$ absolute difference, and were of no statistical significance $(P>$ $0.30)$.

\section{DISCUSSION}

In this study, we proposed that the bcSUVR and the RE plot improve the estimates of DVR from SUVR and the Logan plot, respectively. While the bcSUVR allows simplification of ${ }^{11} \mathrm{C}-\mathrm{PiB}$ dynamic PET data acquisition and quantification, the RE plot needs to be applied to the full dynamic PET data. SUVR is associated with positive bias observed with ${ }^{11} \mathrm{C}-\mathrm{PiB} \quad(13,15)$ and with neuroreceptor ligands $(17,35,36)$. Although this bias has been analyzed by compartmental modeling techniques $(16,17)$, so far no approaches have been developed for mitigating such bias. The recently developed relative equilibrium-based graphical plot, the RE plot, was used to derive a correction factor that is able to mitigate the known positive bias associated with SUVR (25). Because the method for bcSUVR is based on the parameters of $\lambda$ and $\mu$ estimated from a population, the generalizability of the method was validated by a cross-validation study within our dataset (supplemental data).

Quantification of the full dynamic PET dataset can also lead to inconsistent bias, as demonstrated by the DVR 


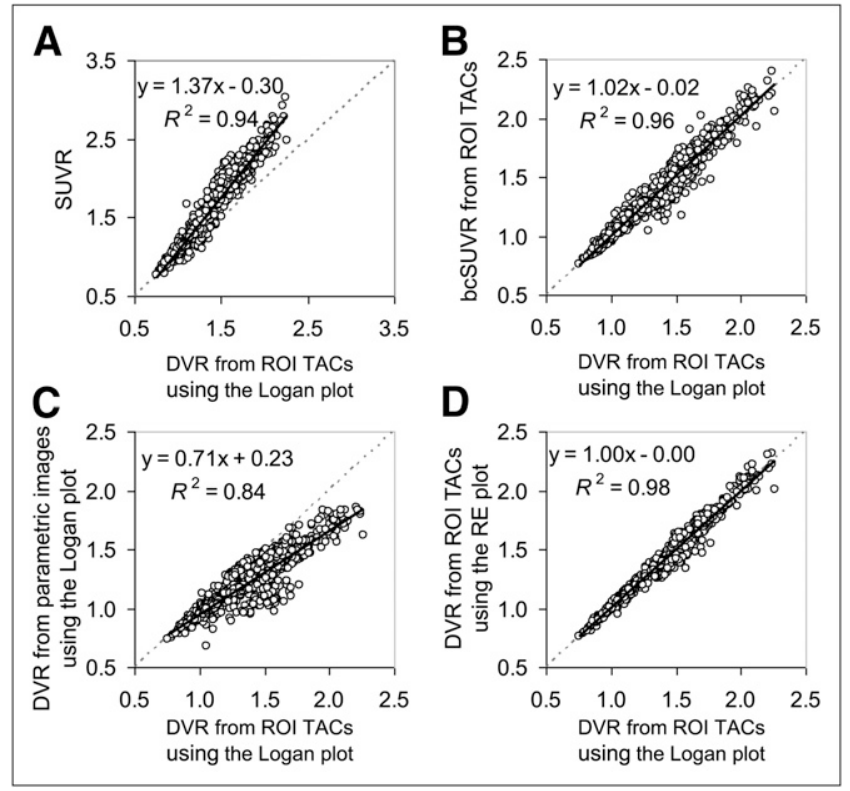

FIGURE 2. Linear correlations between DVR estimates from ROI time-activity curves using Logan plot and SUVR (A), bcSUVR (B), DVR from parametric images generated by Logan plot (C), and DVRs from parametric images generated by RE plot (D). TAC = time-activity curve.

images generated by the Logan plot. Noise-induced underestimation is also observed in the DVR images generated by the SRTM with the conventional multilinear regression method (24). In fact, for ROI ${ }^{11} \mathrm{C}-\mathrm{PiB}$ kinetics of low noise levels, it was reported that the SRTM, 2-parameter SRTM, multilinear reference tissue model 2, and Logan plot provide similar DVR estimates $(10,14)$. In this study, we also found that the DVRs from the SRTM with ROI kinetics were as close to the DVRs from the RE plot as $\operatorname{DVR}($ SRTM with ROI time-activity curves $)=1.03 \mathrm{DV}(\mathrm{RE}$ plot $)-0.07\left(R^{2}=0.96\right)$.

Both the RE plot and the bcSUVR, as well as SUVR, require the presence of equilibrium relative to reference tissue kinetics for $\mathrm{t} \geq \mathrm{t} *$. Because it may take longer to achieve relative equilibrium for tissues with lower clearance rates or higher DVRs, the $t *$ is likely to be determined by the ROI kinetics from older subjects and MCI patients.
Here, we have validated that $t^{*}$ occurs at 52.5 min after tracer injection in populations including MCI subjects and controls with age ranges from 77.2 to $89.5 \mathrm{y}$ and a maximum ROI DVR as high as 2.265. The onset of the relative equilibrium $\mathrm{t}^{*}$ at $40-50 \mathrm{~min}$ was demonstrated in other ${ }^{11} \mathrm{C}$ $\mathrm{PiB}$ studies, including those with controls and $\mathrm{MCI}$ and $\mathrm{AD}$ patients $(12,13,15)$. As such, $t^{*}=52.5$ min corresponding to dynamic PET scan frames starting from 50 min after tracer injection is an appropriate value for the RE plot and bcSUVR estimation of DVR in ${ }^{11} \mathrm{C}-\mathrm{PiB}$ PET studies. Relative equilibrium tracer kinetics were also observed in ${ }^{11} \mathrm{C}$ raclopride for dopamine $\mathrm{D}_{2}$-like receptor imaging $(17,25)$, 2-(1-\{6-[(2- ${ }^{18} \mathrm{~F}$-fluoroethyl)(methyl)amino]-2-naphthylethylidene) malononitrile $\left({ }^{18} \mathrm{~F}\right.$-FDDNP) for imaging $\beta$-amyloid plaques and neurofibrillary tangles (37), and ${ }^{18} \mathrm{~F}$-florbetapir and ${ }^{18} \mathrm{~F}$-florbetaben for imaging $\beta$-amyloid $(38,39)$. As such, the RE plot and bcSUVR methods are applicable to these tracer kinetics.

One limitation of this population-based approach is that the correction factors $\lambda$ and $\mu$ obtained here may not be applicable to different populations such as AD patients or to data acquired on different scanners using different acquisition or image reconstruction protocols. However, once the bcSUVR method is carefully validated using the RE plot on the full dynamic PET scan dataset of a population of interest, it can then be used in simplified PET protocols with short acquisitions applied to individuals from that population.

\section{CONCLUSION}

We have theoretically analyzed inconsistent overestimation of DVR in SUVR and the noise-induced underestimation of DVR by the Logan plot and then demonstrated them on ${ }^{11} \mathrm{C}-\mathrm{PiB}$ studies of nondemented older adults. We propose that bcSUVR derived from the RE plot can simplify both clinical and research ${ }^{11} \mathrm{C}-\mathrm{PiB}$ data quantification. The RE plot and bcSUVR are associated with low bias, high consistency, and high computational efficiency in quantification of ${ }^{11} \mathrm{C}-\mathrm{PiB}$ retention. Both of these methods improve the quantification of DVR estimates compared with the Logan plot and the SUVR, 2 frequently used methods for assessment of $\beta$-amyloid burden.

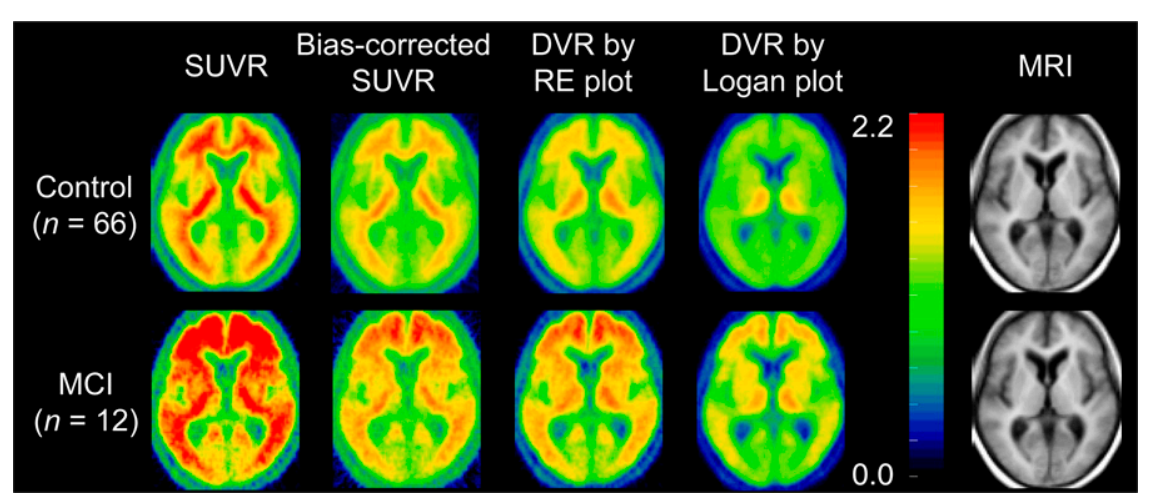

FIGURE 3. Mean of parametric images generated using SUVR, bcSUVR, and DVR images generated by RE plot and Logan plot in $\mathrm{MCl}$ and control groups. Mean MR image is provided for anatomic information. 


\section{DISCLOSURE STATEMENT}

The costs of publication of this article were defrayed in part by the payment of page charges. Therefore, and solely to indicate this fact, this article is hereby marked "advertisement" in accordance with 18 USC section 1734.

\section{ACKNOWLEDGMENTS}

We thank the cyclotron staff, PET staff, and MRI staff of the Johns Hopkins Medical Institutions and Andrew H. Crabb for data transfer and computer administration. This study was supported in part by the Intramural Research Program of the NIH and by the National Institute on Aging (NIA) (R\&D contracts N01-AG-0012 and N01-AG-3-2124). No other potential conflict of interest relevant to this article was reported. This work was presented in part at the 56th Society of Nuclear Medicine conference, June 13-17, 2009, Toronto, Canada, and at the 57th Society of Nuclear Medicine conference, June 5-9, 2010, Salt Lake City, Utah.

\section{REFERENCES}

1. Vallabhajosula S. Positron emission tomography radiopharmaceuticals for imaging brain Beta-amyloid. Semin Nucl Med. 2011;41:283-299.

2. Ziolko SK, Weissfeld LA, Klunk WE, et al. Evaluation of voxel-based methods for the statistical analysis of PIB PET amyloid imaging studies in Alzheimer's disease. Neuroimage. 2006;33:94-102.

3. Kemppainen NM, Aalto S, Wilson IA, et al. PET amyloid ligand $\left[{ }^{11} \mathrm{C}\right] \mathrm{PIB}$ uptake is increased in mild cognitive impairment. Neurology. 2007;68:1603-1606.

4. Kemppainen NM, Aalto S, Wilson IA, et al. Voxel-based analysis of PET amyloid ligand $\left[{ }^{11} \mathrm{C}\right] \mathrm{PIB}$ uptake in Alzheimer disease. Neurology. 2006;67:1575-1580.

5. Aizenstein HJ, Nebes RD, Saxton JA, et al. Frequent amyloid deposition without significant cognitive impairment among the elderly. Arch Neurol. 2008;65:1509-1517.

6. Innis RB, Cunningham VJ, Delforge J, et al. Consensus nomenclature for in vivo imaging of reversibly binding radioligands. J Cereb Blood Flow Metab. 2007;27:1533-1539.

7. Koeppe RA, Holthoff VA, Frey KA, Kilbourn MR, Kuhl DE. Compartmental analysis of $\left[{ }^{11} \mathrm{C}\right]$ flumazenil kinetics for the estimation of ligand transport rate and receptor distribution using positron emission tomography. J Cereb Blood Flow Metab. 1991;11:735-744.

8. Logan J, Fowler JS, Volkow ND, et al. Graphical analysis of reversible radioligand binding from time-activity measurements applied to $\left[\mathrm{N}-{ }^{11} \mathrm{C}\right.$-methyl]-(-)-cocaine PET studies in human subjects. J Cereb Blood Flow Metab. 1990;10:740-747.

9. Lammertsma AA, Hume SP. Simplified reference tissue model for PET receptor studies. Neuroimage. 1996;4:153-158.

10. Zhou Y, Resnick SM, Ye W, et al. Using a reference tissue model with spatial constraint to quantify $\left[{ }^{11} \mathrm{C}\right]$ Pittsburgh compound B PET for early diagnosis of Alzheimer's disease. Neuroimage. 2007;36:298-312.

11. Logan J, Fowler JS, Volkow ND, Wang GJ, Ding YS, Alexoff DL. Distribution volume ratios without blood sampling from graphical analysis of PET data. J Cereb Blood Flow Metab. 1996;16:834-840.

12. Price JC, Klunk WE, Lopresti BJ, et al. Kinetic modeling of amyloid binding in humans using PET imaging and Pittsburgh Compound-B. J Cereb Blood Flow Metab. 2005;25:1528-1547.

13. Lopresti BJ, Klunk WE, Mathis CA, et al. Simplified quantification of Pittsburgh Compound B amyloid imaging PET studies: a comparative analysis. J Nucl Med. 2005;46:1959-1972.

14. Yaqub M, Tolboom N, Boellaard R, et al. Simplified parametric methods for $\left[{ }^{11} \mathrm{C}\right] \mathrm{PIB}$ studies. Neuroimage. 2008;42:76-86.

15. McNamee RL, Yee SH, Price JC, et al. Consideration of optimal time window for Pittsburgh compound B PET summed uptake measurements. J Nucl Med. 2009; 50:348-355.
16. Carson RE, Channing MA, Blasberg RG, et al. Comparison of bolus and infusion methods for receptor quantitation: application to $\left[{ }^{18} \mathrm{~F}\right]$ cyclofoxy and positron emission tomography. J Cereb Blood Flow Metab. 1993;13:24-42.

17. Logan J, Alexoff D, Kriplani A. Simplifications in analyzing positron emission tomography data: effects on outcome measures. Nucl Med Biol. 2007;34:743756.

18. Wallius E, Nyman M, Oikonen V, Hietala J, Ruotsalainen U. Voxel-based NK1 receptor occupancy measurements with $\left[{ }^{18} \mathrm{~F}\right] \mathrm{SPA}-\mathrm{RQ}$ and positron emission tomography: a procedure for assessing errors from image reconstruction and physiological modeling. Mol Imaging Biol. 2007;9:284-294.

19. Slifstein M, Laruelle M. Effects of statistical noise on graphic analysis of PET neuroreceptor studies. $J$ Nucl Med. 2000;41:2083-2088.

20. Logan J, Fowler JS, Volkow ND, Ding YS, Wang GJ, Alexoff DL. A strategy for removing the bias in the graphical analysis method. J Cereb Blood Flow Metab. 2001;21:307-320.

21. Fujimura Y, Ikoma Y, Yasuno F, et al. Quantitative analyses of ${ }^{18} \mathrm{~F}$-FEDAA1106 binding to peripheral benzodiazepine receptors in living human brain. $J \mathrm{Nucl}$ Med. 2006;47:43-50.

22. Abi-Dargham A, Martinez D, Mawlawi O, et al. Measurement of striatal and extrastriatal dopamine D1 receptor binding potential with $\left[{ }^{11} \mathrm{C}\right] \mathrm{NNC} 112$ in humans: validation and reproducibility. J Cereb Blood Flow Metab. 2000;20: $225-243$.

23. Ichise M, Liow JS, Lu JQ, et al. Linearized reference tissue parametric imaging methods: application to $\left[{ }^{11} \mathrm{C}\right] \mathrm{DASB}$ positron emission tomography studies of the serotonin transporter in human brain. J Cereb Blood Flow Metab. 2003;23:10961112 .

24. Zhou Y, Endres CJ, Brasic JR, Huang SC, Wong DF. Linear regression with spatial constraint to generate parametric images of ligand-receptor dynamic PET studies with a simplified reference tissue model. Neuroimage. 2003;18:975-989.

25. Zhou Y, Ye W, Brasic JR, Crabb AH, Hilton J, Wong DF. A consistent and efficient graphical analysis method to improve the quantification of reversible tracer binding in radioligand receptor dynamic PET studies. Neuroimage. 2009;44:661-670.

26. Resnick SM, Goldszal AF, Davatzikos C, et al. One-year age changes in MRI brain volumes in older adults. Cereb Cortex. 2000;10:464-472.

27. Morris JC, Storandt M, Miller JP, et al. Mild cognitive impairment represents early-stage Alzheimer disease. Arch Neurol. 2001;58:397-405.

28. Driscoll I, Resnick SM, Troncoso JC, An Y, O'Brien R, Zonderman AB. Impact of Alzheimer's pathology on cognitive trajectories in nondemented elderly. Ann Neurol. 2006;60:688-695.

29. Kawas C, Gray S, Brookmeyer R, Fozard J, Zonderman A. Age-specific incidence rates of Alzheimer's disease: the Baltimore Longitudinal Study of Aging. Neurology. 2000;54:2072-2077.

30. Cunningham VJ, Jones T. Spectral analysis of dynamic PET studies. J Cereb Blood Flow Metab. 1993;13:15-23.

31. Lawson C, Hanson RJ. Solving Least Squares Problem. Englewood Cliffs, NJ: Prentice Hall; 1974

32. Zhou Y, Ye W, Brasic JR, et al. Spectral analysis with a minimal basis function approach for quantification of ligand-receptor dynamic PET study. J Cereb Blood Flow Metab. 2005;25:S634.

33. Akaike H. An information criteria (AIC). Math Sci. 1976;14:5-9.

34. Turkheimer FE, Hinz R, Cunningham VJ. On the undecidability among kinetic models: from model selection to model averaging. J Cereb Blood Flow Metab. 2003;23:490-498.

35. Carson RE. PET physiological measurements using constant infusion. Nucl Med Biol. 2000;27:657-660.

36. Slifstein M. Revisiting an old issue: the discrepancy between tissue ratio-derived binding parameters and kinetic modeling-derived parameters after a bolus of the serotonin transporter radioligand ${ }^{123}$ I-ADAM. J Nucl Med. 2008;49:176-178.

37. Wong K, Huang S, Kepe V, Small GW, Barrio JR. Evaluation of two graphical approaches for regional analysis and parametric mapping of dynamic $\left[{ }^{18} \mathrm{~F}\right]$ FDDNP PET studies. IEEE Nucl Sci Symposium Conf Rec. 2009:3946-3949.

38. Rowe CC, Ackerman U, Browne W, et al. Imaging of amyloid beta in Alzheimer's disease with ${ }^{18} \mathrm{~F}-\mathrm{BAY} 94-9172$, a novel PET tracer: proof of mechanism. Lancet Neurol. 2008;7:129-135.

39. Wong DF, Rosenberg PB, Zhou Y, et al. In vivo imaging of amyloid deposition in Alzheimer disease using the radioligand ${ }^{18} \mathrm{~F}-\mathrm{AV}-45$ (florbetapir [corrected] F 18). J Nucl Med. 2010;51:913-920. 